\title{
Foreshortened tiles in paths converging on an observer viewing a picture: Elevation and visual angle ratio determine perceived size
}

\author{
Igor JuriceVic, John M. Kennedy, And Izabella Abramov \\ University of Toronto, Toronto, Ontario, Canada
}

\begin{abstract}
Observers were shown wide-angle pictures of tiles on a ground plane and were asked about the aspect ratios of the tiles. The observers viewed the pictures from a fixed center of projection. Some of the tiles were in a path coming straight toward the observer. In one picture, the path came from the center of the picture, and in two others the path came from the left side of the picture (one from $30^{\circ}$ and one from $45^{\circ}$ to the left of the center, from the observer's point of view). The apparent aspect ratios were a function of the elevations of the tiles and the ratios of visual angles of the sides of the tiles. Judgments were identical for all three paths. The local slant of the picture surface was not a significant factor.
\end{abstract}

Most people who view perspective pictures are probably unaware that such pictures can result in severely distorted views of the 3-D world that is depicted. Knowledge of perspective distortions is important for many reasons. For one, perspective pictures and photographs are often freely substituted for real scenes in many applications and experiments, even though their perceptual functions are poorly understood.

We have been engaged in a program of research to explore some of the problematic conditions that are encountered in picture perception, and we are reporting here an experiment involving wide-angle panoramic pictures. Specifically, we depicted a tiled ground plane in a wideangle view, $45^{\circ} \times 105^{\circ}$ (Figure 1 ). The perceived aspect ratio (i.e., the ratio of perceived depth to perceived width) of the depicted tiles - that is, the tiles in the "real world" that is shown in the picture-varied widely. We assessed the influence of tile elevation at the observer's vantage point (an angle, related to the vanishing point, that is sometimes described loosely as "height," in the picture), of the direction of a path of tiles (oblique or straight ahead, which changes the slant of the surface that is depicting the tile), and of the angular measures of the shape of the tile (the aspect ratio of the visual angles of the tiles).

The apparent distortions that we report are systematic and are related to the visual geometry. In brief, what matters is a tile's elevation and the angular measures of its shape, not path direction.

Elevation is an angular measure. A zero elevation $\left(0^{\circ}\right)$ means that a tile is at the observer's feet. As tiles that are on flat ground approach the horizon, they approach an eleva- tion of $90^{\circ}$. A tile that is on flat ground and is at a distance from an observer that is equal to the observer's eye height is at an elevation of $45^{\circ}$. Hence, elevation is related to distance (Cutting, 2003, p. 229; Sedgwick, 2003, p. 69).

Elevation is related to projected shape as well as to distance. A square tile that is at the observer's feet has an elevation of $0^{\circ}$, and all its sides subtend equal angles at the eye. If the tile approaches the horizon, its sides project smaller and smaller angles, and the angles that are subtended by the sides that are running directly away from the observer are especially foreshortened. Hence, elevation affects the ratios of the angles that are projected by the sides that represent depth and width. The ratios provide information about the distant tile's shape.

Elevation matters to angles that are subtended and to information about shape. Goodman (1968, pp. 10-19) noted that elevation is of interest to perception and relates to projected shape as well as to depth. He noted that pictures of tall buildings could show that sides converge as elevation increases, just as pictures of railroad tracks have lines that converge with distance. Convergence occurs because the picture plane is tilted with respect to the façade of the building or to the railroad tracks (Kubovy, 1986, pp. 122-126).

Information about the shape of an object is provided by the visual angles of its parts and by their ratios. Gibson (1950) pointed out that invariant ratios would be useful in theories of visual constancy. Vision might pick out the invariant ratio and use it to see a constant shape despite changes that are due to foreshortening with distance. Sides of tiles that are on the ground at the observer's feet 


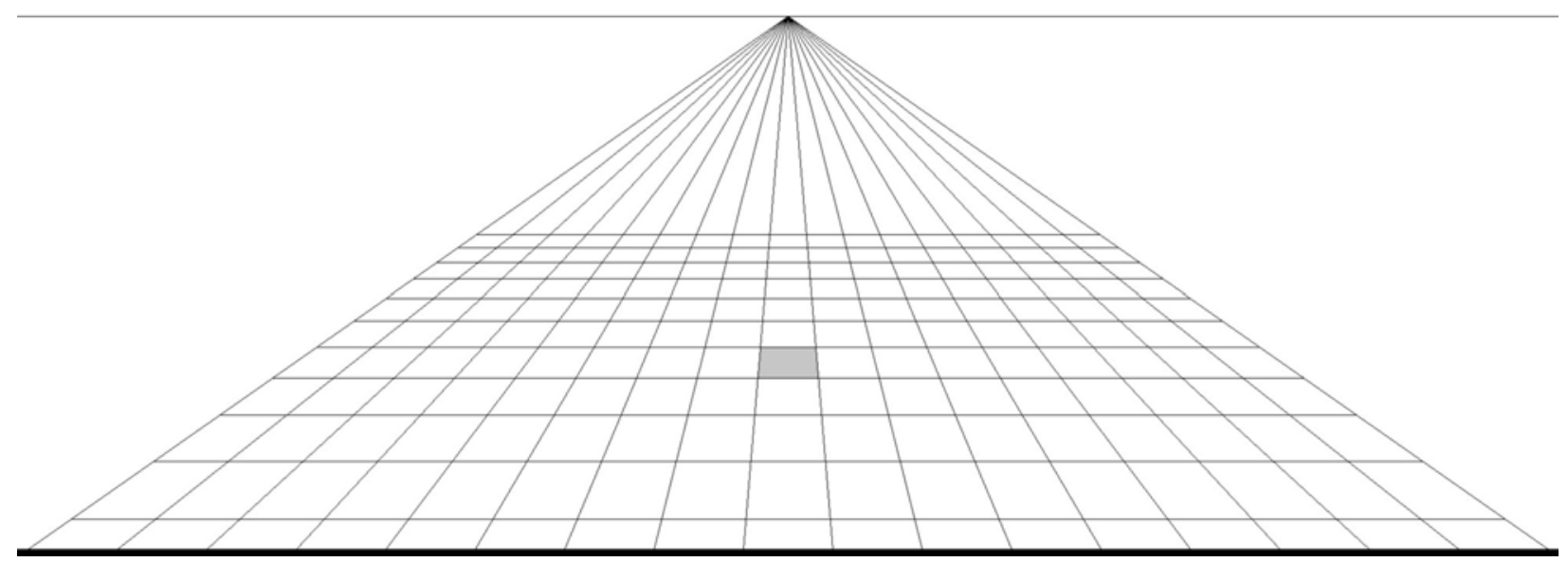

Figure 1. Perspective picture of a piazza of square tiles, with parallel paths of tiles receding at right angles to the picture surface. The $x$ (or width) dimension is given by the horizontals and the $z$ (or depth) dimension is given by the height of the tiles on the picture surface. The picture subtended $45^{\circ} \times 105^{\circ}$ at the observer's viewpoint.

subtend equal angles at the observer's vantage point, however, and foreshortening with distance and elevation affects the subtense of sides that are running directly away from the observer especially strongly. In this case, fixed ratios of angles are not likely to be useful for producing shape constancy. We argue that shape constancy may require a function that includes elevation as well as angular ratios of sides, not just an invariant angular ratio that is projected by the parts of the object.

That path direction did not matter in the monocular conditions here is of special interest, because the local slants of the picture surface varied across paths. In contrast, local slant affects picture-viewing in close-up binocular conditions. Vishwanath, Girshick, and Banks (2005) tested perspective pictures that were viewed obliquely in binocular conditions and found that perception of forms was affected by the slant of the picture's surface as well as the geometric information that depicted the object. The effects of slant assisted in the production of shape constancy. Our viewing conditions were monocular, however, simulating the viewing of distant sources and omitting the spatial information that binocular viewing provides (Sedgwick, 2003, pp. 65-66).

Our report matters to a classic problem: understanding visual reactions to angular projections from distal objects (Gibson, 1950). We test linear-perspective pictures that were viewed from a single center of projection. Geometrically, each picture was a projection of tiles with a given aspect ratio. We employed wide-angle pictures, a feature of our work that calls for explanation. Wide-angle pictures are not common, precisely because they have the potential for perspective distortion (Kubovy, 1986). Their oddities have been known since the Renaissance. Piero della Francesca argued that, to avoid distortions, the picture should subtend less than $90^{\circ}$ - that is, less than $45^{\circ}$ on either side of the line that runs directly from the observer to the center of the picture (della Francesca, c. 1480/1981). Beyond $45^{\circ}$, the "depth," or $z$ dimension, of square tiles projects lines that are longer than the "width," or $x$ dimension (Ken- nedy \& Juricevic, 2002). Leonardo da Vinci similarly recommended that the proportions of perspective pictures be severely restricted. He advised that the distance from the center of projection (spectator point) to the picture plane be more than 10 times the height or length of the principal object (Kubovy, 1986). Many painters since Leonardo have constructed wide-angle-perspective pictures that break his rule, but often the artists have carefully obscured any floor tiles that were likely to appear distorted by hiding them behind foreground objects such as pillars, trees, or people. Juricevic and Kennedy (2006) suggested that vision uses an approximation to perspective that fits Leonardo's rule for narrow widths, and that this approximation produces apparent distortions when one views wide-angle pictures. That is, wide-angle pictures and the distortions that they produce reveal visual processes. Hence, measurements of the distortions that are apparent in wide-angle pictures can test proposals about vision's approximation to correct perspective. (Since real scenes project to the eye according to perspective principles, furthermore, tests of the distortions that are seen in wide-angle pictures may help us understand perception of real scenes as well as of pictures.)

From first principles, the observer who is judging the aspect ratio of tiles on a ground plain should use two factors: The first is angular elevations of tiles. One can define this factor with respect to a vanishing point on the horizon of the plain. The second is the visual angle ratios of the sides. The apparent aspect ratios should be predicted by, but differ from, the visual angle ratios.

Consider observers on a ground plane. If they are standing on a square tile, its sides subtend equal angles from their vantage point. If the square tile is one of a set of identical tiles that lie in a long path that stretches toward the horizon, the projections of tiles that are close to the horizon are highly foreshortened at their vantage point. The angular ratio of the $x$ dimension of the tiles in the center path in Figure 1 to their orthogonal dimension approaches a limit of zero as the tiles approach the horizon. Despite perspective, observers often perceive 


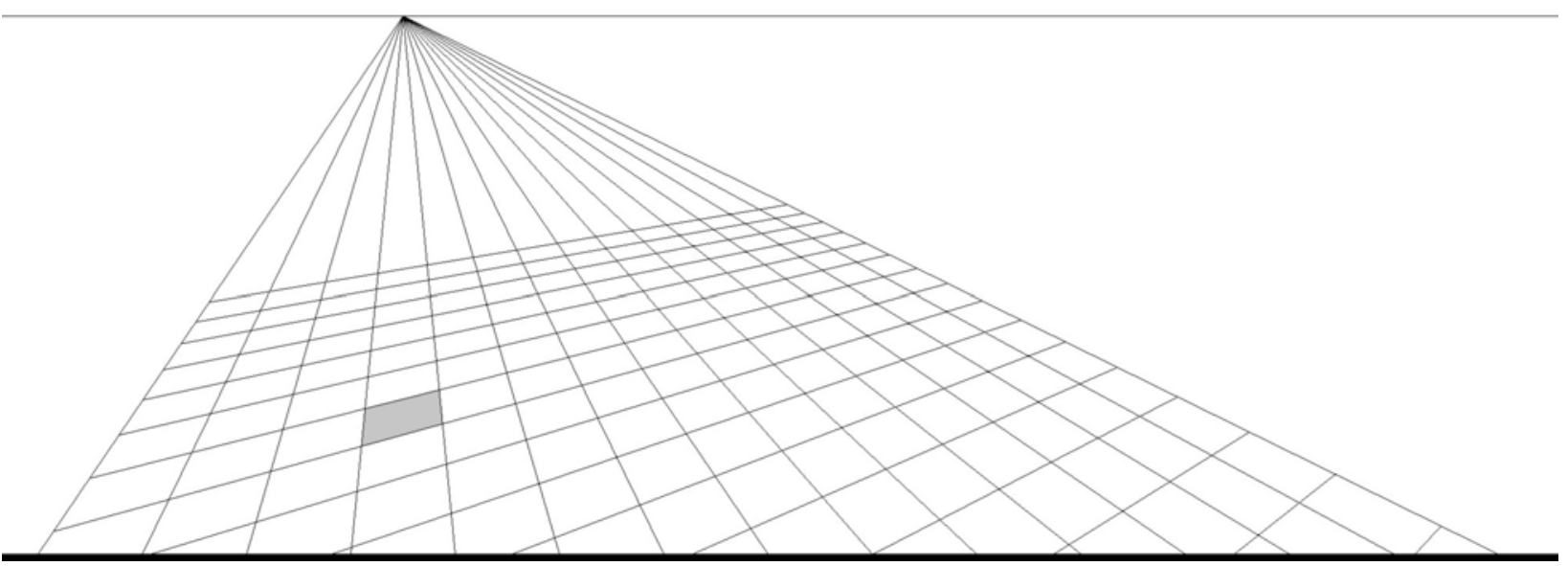

Figure 2. Perspective picture with paths of square tiles, with the path that contains the shaded tile receding at $30^{\circ}$ from the normal to the picture surface. The shaded tile in Figure $\mathbf{2}$ is located at the same distance from the observer as that for the shaded tile in Figure 1.

the size and shape of some parts of the scene to be approximately constant. For example, several of the middistance tiles in the center of the picture might be seen as approximately square in spite of differences in foreshortening (Juricevic \& Kennedy, 2006). This is called shape constancy. Tiles on the side of the picture are often seen as elongated, however, in what is called marginal distortion (Kubovy, 1986) or apparent forelengthening (Kennedy \& Juricevic, 2002). Conversely, tiles in the top rows might appear short and stubby (Juricevic \& Kennedy, 2006, Figure 4D; Rogers, 2003).

Studying foreshortening, Juricevic and Kennedy (2006) asked observers to judge shapes in wide-angle-perspective pictures of a ground plane that was covered with square tiles. The observers reported the apparent aspect ratios of the tiles - that is, the $z$ dimension divided by the $x$ dimension. In keeping with the severe foreshortening that occurs near the vanishing point, the perceptual significance of a given aspect ratio changed with the direction of the tile with respect to the vanishing point. A small ratio far from the vanishing point meant a slim tile, but near the vanishing point it meant a larger tile. The effect of the local slant of the picture surface was not tested.

What is novel in the present study is that we have considered the aspect ratios of an important subset of the tiles in wide-angle pictures - tiles on paths that stretch directly away from the observer (Goldstein, 1987). We describe these as being on straightaway paths. If an observer is standing on several paths that stretch straightaway from the observer's vantage point, the paths radiate from the observer (or converge toward the observer). In one picture, the straightaway path was in the center of the picture (Figure 1). In another, a path approached the observer $30^{\circ}$ from the left of center (Figure 2), and in the third it approached $45^{\circ}$ from the left of center (Figure 3). Observers were placed at the center of the projection that was used to create the figures,

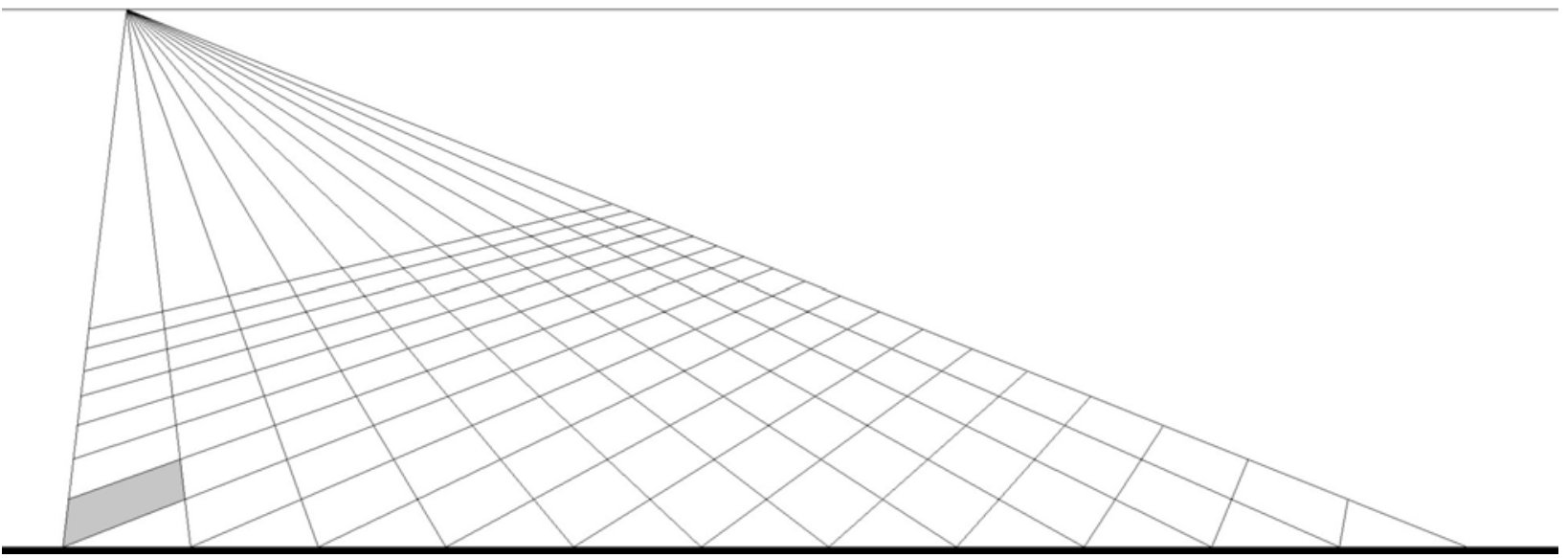

Figure 3. Perspective picture with paths of square tiles, with the path that contains the shaded tile receding at $45^{\circ}$ from the normal to the picture surface. The shaded tile in Figure 3 is located at the same distance from the center of projection as that for the shaded tiles in Figures 1 and 2. The appearance of the aspect ratio of the tiles varies if the reader is not observing any of the three pictures from the center of projection. 


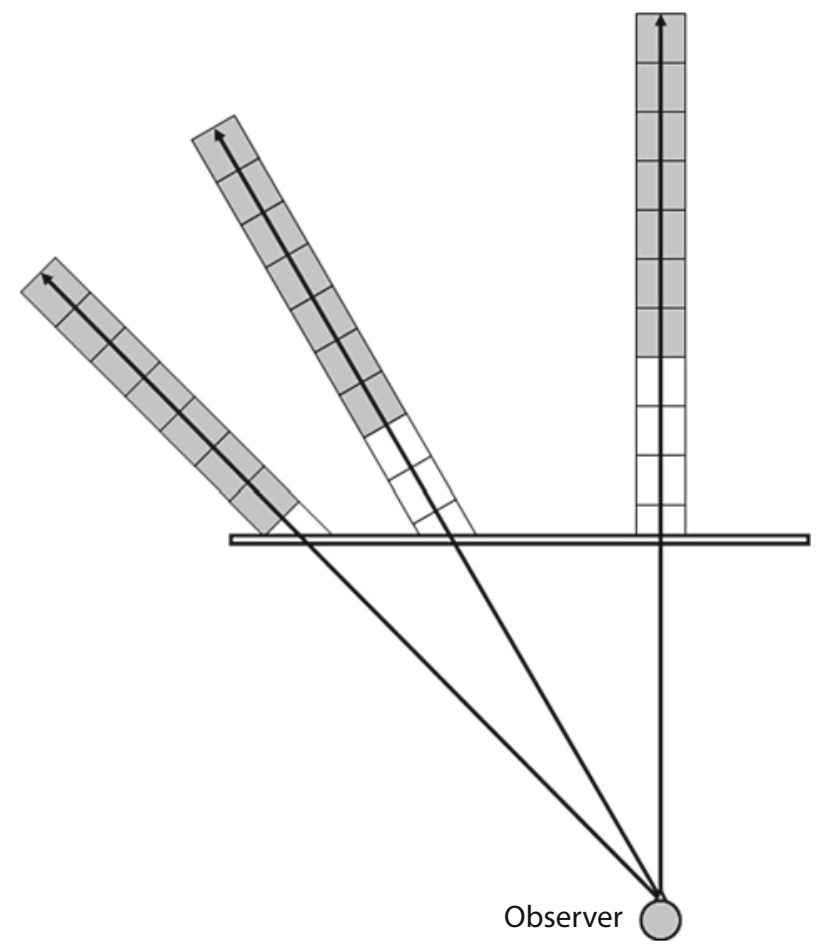

Figure 4. Bird's-eye view of the paths of tiles that are depicted as diverging from the center of projection. The paths radiate from the observer.

and they viewed it monocularly. Figure 4 is a bird's-eye view of the straightaway paths in Figures $1-3$.

The paths in Figures 1-3 have very useful features. They distinguish a tile's elevation from the local orientation of the picture surface. In Figures 2 and 3, the dark tiles are projected on the side of the picture to a greater extent than they are in Figure 1. Looked at from the center of projection, they are on pictorial surfaces with different local slants. Viewed from the center of projection, however, each path has tiles with the same elevations. In addition, Figures 1-3 conveniently distinguish the directions of vanishing points. The vanishing point for Figure 1 is in the center of the picture, whereas in Figures 2 and 3 it is to the left. Geometrically, each receding "column," or path of tiles, in Figures 1-3 heads in the direction of its own vanishing point, and in Figures 2 and 3 the vanishing point is to the left of center. This design for the pictures is helpful in removing a conventional confound with picture composition, since the vanishing point in perspective pictures is often centered and located on the point of the picture surface that is nearest to the observer. That point is at the foot of the "normal" from the observer to the picture plane, the line from the center of projection that is orthogonal to the picture. Additionally, the figures help one to distinguish the shape on the picture surface and the visual angles of the sides of the tiles. The symmetrical dark trapezium in Figure 1 becomes asymmetrical in Figure 2 and highly asymmetrical in Figure 3, but at the center of projection the visual angles that are subtended by the sides of each of the three dark tiles are identical. Finally, the projections of the lines on the picture surface have aspect ratios that are different from their visual angle ratios. The projected lines for the side and base of the tile at an elevation of $45^{\circ}$ have an aspect ratio close to 1.0. Closer to the horizon, however, the lines for the side of the tile subtend smaller angles than does the baseline, with their visual angle ratio reaching 0 at the limit. As tiles elevate from $45^{\circ}$, the discrepancy between projected-line aspect ratio and visual-angle ratio diminishes: The higher the elevation of the central path in Figure 1, the closer a tile in part of the picture surface that contains base- and side lines is to orthogonal to the normal from the center of projection.

Perception of the eccentric tiles might be strongly affected by local surface orientation. Alternatively, perception of the tiles might be invariant across the three paths and related to the elevations of the tiles - their directions with respect to their vanishing points. A third possibility is that the perception of each tile might be determined mostly by the ratios of the lengths of the lines it projects on the picture surface. In the main, ratios of line lengths seem unlikely to be the dominant factor, since viewers take small patches on picture surfaces to mean distant mountains, but they take large patches in the same picture to mean small people. Also, the same line lengths can lead to different perceived aspect ratios when the observer views the picture from different distances (Juricevic \& Kennedy, 2006). A fourth possibility is that a tile's perceived aspect ratio might equal the aspect ratio of its retinal projection. Again, the influence is not likely to operate as a simple ratio, because the visual ratio of mountain and people patches is often the reverse of the ratio of their linear-size judgments. A fifth possibility is more intriguing: Tiles might be judged as invariant in the middle of the path but rapidly changing at the margins. That is, a "window" of tiles might be perceived as invariant. Arguments for a window within which constancy operates were advanced by Kubovy (1986) and considered by Kerzel and Hecht (1997). Finally, the perception of the tiles might be an approximation to linear perspective, but not quite as perspective geometry dictates. Vision's approximation to perspective may underestimate the foreshortening that occurs with distance (Juricevic \& Kennedy, 2006; Rogers, 2003; Smallman, Manes, \& Cowen, 2003). That is, even if a mid-distance, lower row tile in Figure 1 appears to be square, the farther off, higher elevation tiles are likely to appear stubby. For example, a tile that is higher in the picture plane than the dark tile in Figure 1 might appear to have the aspect ratio of a brick, with its long axis in the $x$ dimension.

In Figure 1, the distance from the center of the horizon line to the center of projection is 0.42 times the length of the dark bar at the base of the figures (i.e., the total width of the picture). The stimulus that was based on Figure 1 was calculated to be a projection of square tiles with a center of projection that was $0.54 \mathrm{~m}$ from the picture plane. Mathematically, however, it is also a projection of tiles with a width of 1 unit, a depth of 0.5 , and a center of projection at $1.08 \mathrm{~m}$, double the $0.54-\mathrm{m}$ distance (Cutting, 2003; Kennedy \& Juricevic, 2002). There is no single correct vantage point or tile aspect ratio, although the two are 
related. As a result, increases in the size of the vantage point's distance lead to proportional decreases in tile aspect ratio. Creation of a perspective picture is unambiguous, but once it has been produced, a given projection is ambiguous about the aspect ratios in the scene that it represents. Its "inverse projection" requires that further dimensions be stipulated. These can come from knowledge of viewing distance or an observer's biases, or from rules of thumb. Indeed, Rogers (2003) reported that distances along a ground in pictured scenes were judged to be $40 \%$ of their real-world length, and half of their apparent length when one viewed the real scene. In practice, most people view pictures from many distances. They do not seek the correct vantage point for the projection. If observers often discount their actual viewing distances to a picture, the physical aspect ratio of tiles in Figures 1-3 would be underdetermined. Our concern in the present study, therefore, is with relative perceived aspect ratios along a path of tiles. Indeed, there is no information in Figures 1-3 about tiles with a fixed aspect ratio that should be viewed from a fixed distance.

In sum, our main concerns in the present study were elevation, visual-angle ratio, and local slant. If the local surface orientation of the picture surface affected an observer's impression of the tile's aspect ratio, the dark tiles in the three paths in Figures 1-3 would differ in apparent aspect ratios when viewed from a single vantage point. The local slant for the dark tile in Figure 1 is closer to the normal from that vantage point than is the case in Figures 2 and 3. Figure 3's dark tile has the most extreme local slant. Alternatively, if the perceived aspect ratios were based exactly on the angular projections of the $x$ and $z$ dimensions, the dark tiles in the three paths in Figures 1-3 would look identical when viewed from this standard vantage point, because the visual angle ratio would be the same for each of the three tiles. Finally, the perceived aspect ratios of the tiles that are in any path may not be based solely on their visual angle ratios, but may also vary with elevation - the variable that specifies distance in the $z$ dimension along a plane-though the function may be an approximation that underestimates the rate of foreshortening with elevation.

\section{METHOD}

\section{Subjects}

Ten first-year undergraduates ( 7 female and 3 male, $M=18.6$ years, $S D=2.7$ ) were recruited from an introductory psychology class. All had normal or corrected-to-normal vision and were unaware of the purpose of the experiment.

\section{Stimuli}

The stimuli were Figures 1-3 and other images based on tiles with physically different aspect ratios; they were observed monocularly. In each trial, observers judged the aspect ratio of one tile, which was indicated by bolding its base and one of its side lines. This task is like judging the proportions of a city block or a child's building block by attending to the base of the block. Similar tasks were used by Rogers (2003) and Sedgwick (2003).

A horizontal bar was positioned $0.54 \mathrm{~m}$ from the picture, parallel to the picture plane, and centered in front of the horizon line.
Seated subjects were asked to keep the end of the bar in contact with their temple, to one side of the viewing eye. This allowed the subjects to turn their heads to view all parts of the picture. The subjects could look up, down, left, and right in order to pick up the optic array at their eye. The retinal image changes as we look around from a point of observation, but the optic array-what the observer is scanning-does not. We used the temple-position bar instead of a peephole, because subjects who are free to move backward and forward when using a large peephole can occupy several vantage points. In addition, small peepholes introduce anomalous effects of accommodation that we wished to avoid (Kubovy, 1986). The pictures were back-projected onto a large, translucent projector screen $(0.64 \mathrm{~m} \times 1.28 \mathrm{~m})$ by an EPSON PowerLite $51 \mathrm{c}$ LCD projector (Model EMP-15, resolution $800 \times 600$ pixels).

Seven tiles were tested from each path being tested. In Figure 3, seven tiles fit between the lower border and the highest border of the path with the shaded tile. This set the standard for creating the other figures. The ends of the horizon line subtended $105^{\circ}$, and the lower border was set at an elevation of $45^{\circ}$. Tiles above $45^{\circ}$ elevation project foreshortened (della Francesca, c. 1480/1981; Kennedy \& Juricevic, 2002). The paths being tested were made of tiles with aspect ratios of $1.00,1.72$, and 2.44 , at the observation distance of $0.54 \mathrm{~m}$. (For Figures 1-3, all of the tiles that were used to generate the pictures were square; that is, their aspect ratio was 1 if the distance from the center of the horizon line to the center of projection was 0.42 times the length of the dark bar at the base of the figures.) Each given path had tiles of a fixed aspect ratio. No path had tiles with a mixture of aspect ratios.

In Figures 1-3, tiles with matched aspect ratios have the same elevations across pictures. For example, the baseline of the lowest row of tiles in Figure 3 has an elevation of $45^{\circ}$. This was the benchmark for the elevation of the lowest tiles that were tested in Figures 1-3. The elevation of the second lowest row depended upon the aspect ratios of the tiles $(1.00,1.72$, and 2.44). The baseline of the row with the largest elevation was at an elevation of $76.7^{\circ}$, which occurred in the pictures of tiles with aspect ratios of 2.44. (An elevation of $45^{\circ}$ can also be described as a declination of $45^{\circ}$ from the path's vanishing point on the horizon, and an elevation of $76.7^{\circ}$ is a declination of $13.3^{\circ}$.) Tiles that were lowest in elevation were the tiles that were closest in distance along the horizontal plain that was depicted. Tiles that were highest in elevation were the tiles that were farthest. Given three paths $\left(0^{\circ}, 30^{\circ}\right.$, and $\left.45^{\circ}\right)$, seven tiles per path, and three aspect ratios, a total of 63 pictures were tested. The pictures were presented in random order for each subject. In each trial, the specific tile that the subject was to judge was highlighted by the use of thicker lines (bolding) for the lower and right edges, as shown in Figure 5.

From the observer's vantage point, the visual angle ratios of corresponding tiles in each path in Figures 1-3 are the same. Within each path, the visual angle ratios of the tiles change with distance, because the visual angle of the $x$ dimension of a tile shrinks at a linear rate as the tile recedes into the distance along the plane, and the $z$ dimension reduces at a quadratic rate. This is why, from the observer's viewpoint, the tiles that are lowest in Figures 1-3 have a visual angle ratio of .45 and the highest tiles (nearest their vanishing points) have a visual angle ratio of .32 .

\section{Procedure}

Subjects were instructed to judge the tiles as if they were looking through a window-judgments should have been made not as if one were measuring the lines on the picture, but rather as if one were viewing the tiles in the real world. They were reminded that the parts of a picture showing a person might be larger than the parts showing a hill, but that the task was to report the relative "real world" size of the hill. The apparent aspect ratio of each tile was to be determined by asking subjects to assume that the closest edge of each tile (e.g., the edge that was represented by a horizontal line in Figure 1) was 100 units, and to judge the length of the edge that receded in depth. If the edge that 


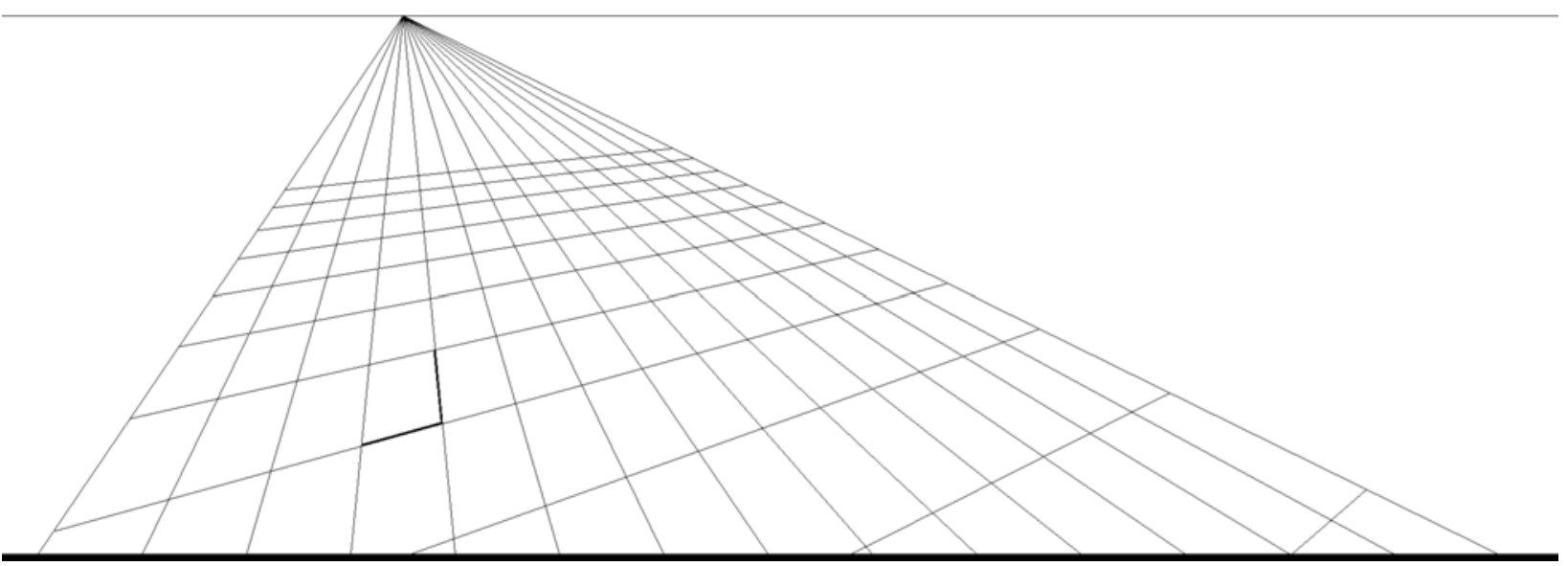

Figure 5. The subjects were asked to judge the aspect ratio of the highlighted tiles. A tile was highlighted by lines that were three times thicker than those that were used to depict the edges.

receded in depth appeared to be larger than the closest edge, then their estimate should have been greater than 100; and if that edge appeared to be shorter, then the estimate should have been smaller than 100 .

\section{RESULTS}

The independent variables for analysis were the three physical aspect ratios of the tiles that were used to generate the stimuli (physical aspect ratio); the seven rows of tiles that extended from low elevation to high (row); and the three diverging paths that were at angles of $0^{\circ}, 30^{\circ}$, and $45^{\circ}$ (path angle).

The dependent variable is the perceived aspect ratio, which in this case was perceived length (the subject's response) divided by perceived width (100 units). A perceived aspect ratio of 1 indicated a square, a perceived aspect ratio that was greater than 1 indicated an "elongated" tile, and a perceived aspect ratio that was less than 1 indicated a "compressed" tile.
Briefly, physical aspect ratio and elevation affected perception, but the three paths were seen as extremely similar. Figure 6 shows that perceived aspect ratio was affected by physical aspect ratio (i.e., of 1.0, 1.72, and 2.44) and row (higher numbered rows indicate higher in elevations).

As is evident in the three distinct response lines for the physical aspect ratios of $1.00,1.72$, and 2.44 , a $3 \times 7 \times 3$ repeated measures ANOVA found that larger aspect ratios were perceived as coming from tiles with larger physical aspect ratios [ physical aspect ratio $F(2,18)=135.5$, $p<.001]$. Bonferroni post hoc tests revealed that the perceived aspect ratios for each of the physical aspect ratios that were used to create the figures differed significantly from one another. Perceived aspect ratio judgments were not completely accurate, but they were compressed by about half compared with the aspect ratios that were used to create the stimuli at the viewing distance, much as was reported by Rogers (2003).

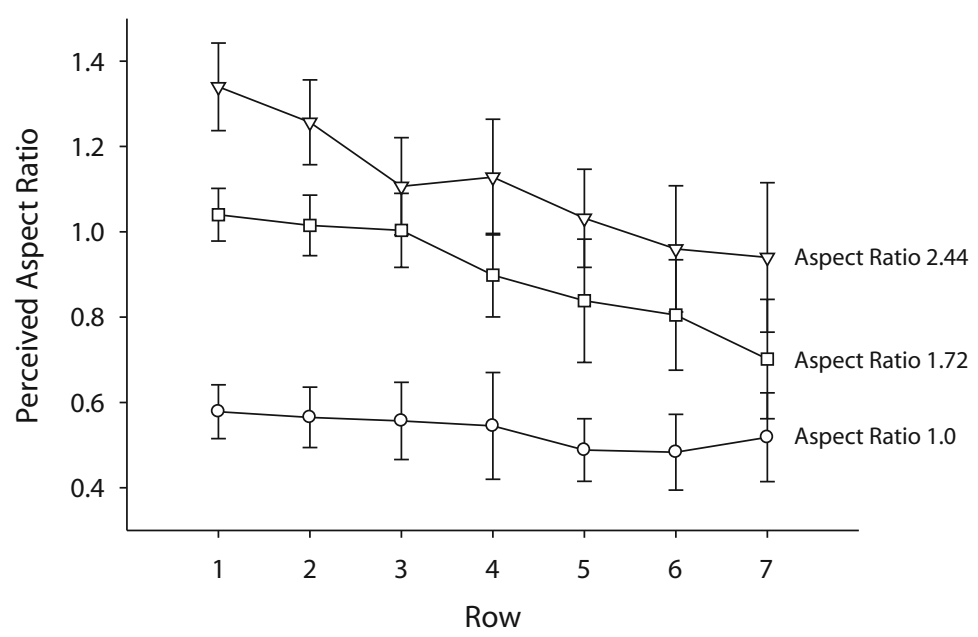

Figure 6. Aspect ratio $\times$ row interaction, with .95 confidence intervals. 
Of importance for the hypothesis that the foreshortening of more distant tiles is underestimated, higher elevation tiles were perceived as smaller $[\operatorname{row} F(6,54)=13.1$, $p<.001]$. Bonferroni post hoc tests revealed that rows 1 and 2 were significantly different from rows 5,6 , and 7 . Judgments of row 3 did not significantly differ from those for any other row. Judgments of row 4 were significantly different from those for row 6 . On average, tiles in the first row were perceived as square, and tiles in the most elevated row were perceived as stubby (an aspect ratio of about 0.7 ; that is, if its width was judged to be 1.0, its depth was only 0.7 ).

Path angle had no main effect $[F(2,18)=0.7, p<$ $.525]$. That is, in each of the paths $\left(0^{\circ}, 30^{\circ}, 45^{\circ}\right)$, the tiles were perceived as being alike, with a mean perceived aspect ratio of about .85 .

Physical aspect ratio $\times$ path angle interaction was not significant $[F(4,36)=0.7, p<.581]$. The path angle $\times$ row interaction was also not significant $[F(12,108)=1.4$, $p<.171]$.

Of importance for the hypothesis that foreshortening in the distance is underestimated, however, is that the physical aspect ratio $\times$ row interaction was significant $[F(12,108)=4.6, p<.001]$, and tiles with larger physical aspect ratios were especially compressed in the more elevated rows. All of the physical aspect ratios showed some diminution with distance (modestly so for the tiles with aspect ratio of 1.0).

The three-way interaction row $\times$ path angle $\times$ physical aspect ratio was not significant $[F(24,216)=0.6, p<$ .992].

\section{DISCUSSION}

The novel aspect of the present method is the use of paths of identical tiles that converge on the observer. The key finding is that the two key variables that influenced perceived tile proportions were visual angle ratio and elevation, but the differences between the paths, with their varying picture-surface local slants, played no role.

Tiles were perceived to be more highly compressed in the higher rows, where visual angle ratios are relatively small and the tiles look like bricks rather than squares.

Path angle $\left(0^{\circ}, 30^{\circ}\right.$, and $\left.45^{\circ}\right)$ made no difference. This finding is interesting, since it leaves only two factors for consideration - visual angle ratio and elevation.

Local-slant information is often obtained from binocular vision, motion parallax, and texture gradients. In binocular vision, picture-surface orientation is used to boost constancy (Vishwanath et al., 2005). This can be helpful in close-up inspection. When one observes an object from afar, texture information can be very helpful. It allows the observer to recognize that a quadrilateral is a square by noticing how it relates to texture units in the scene. For example, it might be four texture units both vertically and horizontally. Sedgwick (2003), however, argues that prominent texture on a picture surface flattens the apparent depth in the scene. If so, it could decrease accuracy. Notice that the texture that is helpful is part of the depicted scene, however, whereas in the case that Sedg- wick described, it is part of the picture surface. Kinetic information is also helpful at large viewing distances. For example, if an observer or camera changes eye height, visual angle ratios of tiles change. The patterns of these changes specify tile aspect ratios. Our concern here has been foreshortening in monocular static vision, however, and in that respect we have no evidence that local surface slant has an impact. It may be instructive for further research to examine binocular, textured, and kinetic conditions under which perception might use local-slant information in wide-angle pictures. It may be that this kind of information for surface slant and the pictured world would decrease the rate at which distortions grow on the margins of wide-angle pictures, which would be helpful in viewing motion pictures from side seats (Cutting, 1987).

The aspect ratios of the tiles that were used to generate the stimuli mattered, since larger aspect ratios were perceived for larger tiles, but the perceived aspect ratios were about half the size of the actual aspect ratios that were used to create the picture at the observation distance, as in Rogers (2003). Observers may have assumed a smaller distance for the center of projection than was actually the case, or used an anchor point that was based on the sample of pictures that were used as stimuli. For example, the mean perceived aspect ratio for tiles in the lowest row was 0.99 , a square. Observers may have used the mean form in the front rows as an anchor for judgments. Subjects frequently view perspective pictures from incorrect vantage points. If vantage-point distance is often in abeyance visually, rules of thumb for accepting an anchor would be useful. In the real world, observers typically have binocular, texture, motion, and other sources of shape information. They can also take into consideration tiles that are directly below their vantage point, at elevation zero. A real square there is specified by a 1:1 visual angle ratio, a highly informative anchor.

The three lines in Figure 6 converge and may be asymptotic at about 0.7 . Observers may have assumed an anchor of 1.0 for the average tile at $45^{\circ}$, and a second anchor for distant tiles. If viewing distance is in abeyance, the absence of specific size and shape information could encourage viewers to adopt such anchors. Between any two anchors there is a rate of change, however. Here, the gradual changes in perceived aspect ratios with elevation indicate that vision was using an approximation to perspective's rate of foreshortening.

Apparent aspect ratio diminished steadily along the paths. The change was gradual, not a sudden transition as if a window of accuracy had abrupt borders. Also, the perceived aspect ratios were not simply the retinal projections - that is, the visual angle ratios. Consider tiles with aspect ratios of 2.44: The visual angle ratios had a range of 0.51 (for the tiles that were highest in elevation) to 1.01 (for the tiles that were lowest in elevation). However, the perceived aspect ratios varied from almost $100 \%$ larger than the visual angle ratio (a perceived aspect ratio of 0.99 for a visual angle ratio of 0.51 ) to $30 \%$ larger than the visual angle ratio (a perceived aspect ratio of 1.31 for a visual angle ratio of 1.01).

Elevation, long recognized as important in depth perception along a ground plane (della Francesca, 
c. 1480/1981; Gibson, 1950; Sedgwick, 2003), influenced the perception of tile proportions. Tiles with a given aspect ratio increased in perceived aspect ratio as their elevation increased. The perceived tiles were neither invariant from the bottom to the top of the paths, nor invariant across paths, as a theory of perfect constancy or correction from local slant would have predicted, and the function that perception used was at best an approximation to perspective.

These results derive from two factors. First, observers do not need to invoke "compensation" for viewing from inappropriate distances (Cutting, 1987; Kerzel \& Hecht, 1997; Yang \& Kubovy, 1999). We tested pictures that were viewed from a center of projection that was on a normal to the center of the figures, not from one side of this standard, which would generate shear. If information about either shear or a proper viewing distance is presented (e.g., by including highly standardized, familiar shapes), compensation might be invoked. Second, although subjects looking at perspective pictures may not normally consider a fixed viewing distance to be essential, the distance might be made relevant by using a mural that appears to extend the observer's ground plane. The pictured ground would be related to the observer's ground, and the viewing distance would become significant.

Our two factors likely influence real-world perception (though with some qualification, since Rogers [2003] found that apparent depth in pictures was less than apparent depth in the world). For example, consider the foreshortening of large, painted arrowheads that are used as direction markers on roads. White arrowheads are often painted on the surface of expressways, pointing sideways to signal an exit. The white-painted arrowhead appears highly acute when first observed from afar, as one drives toward the exit. The arrowhead can appear very sharp, as if the white paint is making an angle of about $10^{\circ}$. In the middle distance, however, the white arrowhead often appears to form a right angle at its tip. When one drives out the exit, finally, a glance straight down reveals that the arrowhead is highly obtuse, perhaps $160^{\circ}$.

The changing perception of the arrowhead angle follows from the underestimation of foreshortened terrain in the distance. Assume that the arrowhead is on a long section of roadway. Let the section have an $x$ dimension of 1 and a $z$ dimension of 20 . The physical aspect ratio (1:20) may be perceived to be quite slight for the $z$ dimension (perhaps 1:0.5). Initially, the sideways-pointing obtuse arrowhead would be severely compressed perceptually in the $z$ dimension, and so would appear highly acute. The $z$ dimension would be perceived more accurately as one approaches the arrowhead.

In sum, in monocular perception of linear perspective pictures, the relation between visual angle ratio and elevation is used as information about a tile's aspect ratios, with no change in the relation because of the local slant of the picture.

\section{AUTHOR NOTE}

This research was supported by grants from NSERC (Canada) and Wissenschaftskolleg zu Berlin to J.M.K. We thank S. Hammad, D. Press, B. Haji, R. Patel, S. Rajani, H. Chan, S. Bhasin, M. Wnuczko, and D. Todorovic. We also thank three reviewers, incuding H. Hecht and M. Kubovy, who kindly suggested the format of Figure 6 and the connection to N. Goodman. Correspondence concerning this article should be addressed to J. M. Kennedy, University of Toronto at Scarborough, 1265 Military Trail, Toronto, ON, M1C 1A4 Canada (e-mail: kennedy@ utsc.utoronto.ca).

Note-Accepted by the previous editorial team, when Thomas H. Carr was Editor.

\section{REFERENCES}

Cutring, J. E. (1987). Rigidity in cinema seen from the front row, side aisle. Journal of Experimental Psychology: Human Perception \& Performance, 13, 323-334

Cutting, J. E. (2003). Reconceiving perceptual space. In H. Hecht, R. Schwartz, \& M. Atherton (Eds.), Looking into pictures: An interdisciplinary approach to pictorial space (pp. 215-238). Cambridge, MA: MIT Press.

Della Francesca, P. (1981). De prospectiva pingendi [On the perspective of painting]. In E. G. Holt (Ed.), A documentary history of art (Vol. 1, pp. 256-267). Princeton, NJ: Princeton University Press. (Original work published 1480)

GiBson, J. J. (1950). The perception of the visible world. Boston: Houghton Mifflin.

Goldstein, E. B. (1987). Spatial layout, orientation relative to the observer, and perceived projection in pictures viewed at an angle. Journal of Experimental Psychology: Human Perception \& Performance, 13, 256-266

Goodman, N. (1968). Languages of art: An approach to a theory of symbols. Indianapolis: Bobbs-Merrill.

JURICEVIC, I., \& KENNEDY, J. M. (2006). Looking at perspective pictures from too far, too close, and just right. Journal of Experimental Psychology: General, 135, 448-461.

Kennedy, J. M., \& JuRICEvic, I. (2002). Foreshortening gives way to forelengthening. Perception, 31, 893-894.

KerZel, D., \& HeCht, H. (1997). Grenzen der perzeptuellen Robustheit bei perspektivischer Verzerrung [The limits of perceptual robustness and perspective distortion]. Zeitschrift für Experimentelle Psychologie, 44, 394-430.

Kubovy, M. (1986). The psychology of perspective and Renaissance art. Cambridge: Cambridge University Press.

Rogers, S. (2003). Truth and meaning in pictorial space. In H. Hecht, R. Schwartz, \& M. Atherton (Eds.), Looking into pictures: An interdisciplinary approach to pictorial space (pp. 301-320). Cambridge, MA: MIT Press.

SEDGWICK, H. A. (2003). Relating direct and indirect perception of spatial layout. In H. Hecht, R. Schwartz, \& M. Atherton (Eds.), Looking into pictures: An interdisciplinary approach to pictorial space (pp. 61-75). Cambridge, MA: MIT Press.

Smallman, H. S., Manes, D. I., \& Cowen, M. B. (2003, October). Measuring and modeling the misinterpretation of 3-D perspective views. Proceedings of the Human Factors and Ergonomics Society 47th Annual Meeting (pp. 1615-1619). Santa Monica, CA: Human Factors and Ergonomics Society.

Vishwanath, D., Girshick, A. R., \& Banks, M. S. (2005). Why pictures look right when viewed from the wrong place. Nature Neuroscience, 8, 1401-1410.

YANG, T., \& KuBOVY, M. (1999). Weakening the robustness of perspective: Evidence for a modified theory of compensation in picture perception. Perception \& Psychophysics, 61, 456-467.

(Manuscript received August 21, 2007; revision accepted for publication September 3, 2008.) 